Association for Information Systems

AIS Electronic Library (AISeL)

Wirtschaftsinformatik 2021 Proceedings

Track 2: General Track - Innovative, emerging and interdisciplinary topics

\title{
Designing an Adaptive Empathy Learning Tool
}

Thiemo Wambsganss

Universität St.Gallen

Florian Weber

Universität Kassel

Matthias Söllner

Universität Kassel

Follow this and additional works at: https://aisel.aisnet.org/wi2021

Wambsganss, Thiemo; Weber, Florian; and Söllner, Matthias, "Designing an Adaptive Empathy Learning Tool" (2021). Wirtschaftsinformatik 2021 Proceedings. 3.

https://aisel.aisnet.org/wi2021/YGeneralTrack/Track02/3

This material is brought to you by the Wirtschaftsinformatik at AIS Electronic Library (AISeL). It has been accepted for inclusion in Wirtschaftsinformatik 2021 Proceedings by an authorized administrator of AIS Electronic Library (AISeL). For more information, please contact elibrary@aisnet.org. 


\title{
Designing an Adaptive Empathy Learning Tool
}

\author{
Thiemo Wambsganss ${ }^{1}$, Florian Weber ${ }^{2}$, and Matthias Söllner ${ }^{2}$ \\ ${ }^{1}$ University of St.Gallen (HSG), Institute of Information Management, St.Gallen, Switzerland \\ thiemo.wambsganss@unisg.ch \\ ${ }^{2}$ University of Kassel, Information Systems and Systems Engineering, Kassel, Germany \\ \{weber, soellner\}@uni-kassel.de
}

\begin{abstract}
Empathy is a fundamental competency for daily communication, interaction, and teamwork, and thus most relevant for future jobs. Nevertheless, educational organizations are limited in providing the necessary conditions for students to develop empathy skills, due to traditional large-scale and distancelearning scenarios. In this paper, we present insights on how to design an adaptive learning tool that helps students to develop their ability to react to other people's observed experiences through individual feedback independent of an instructor, time and location. Based on theoretical insights of 110 papers and 28 user interviews, we propose preliminary design principles for an adaptive empathy learning tool. Moreover, we evaluate the design principles as an instantiated prototype in a proof-of-concept evaluation with 25 students. The results indicate that an empathy learning tool based on the presented design knowledge seems to be a promising approach to help students to improve their empathy skills in different learning scenarios.
\end{abstract}

Keywords: Empathy Learning, Adaptive Skill Learning, Cognitive Dissonance Theory, Design Science Research

\section{Introduction}

"The biggest deficit we have in our society, and in the world right now, is an empathy deficit. We are in great need of people being able to stand in somebody else 's shoes and see the world through their eyes "

\section{Barack Obama in 2009, talking to Students in Istanbul}

As Barack Obama, former president of the United States, stated, empathy is not only an elementary skill for our society and daily interaction but also for professional communication as well as successful teamwork and thus elementary for educational curricula (i.e., Learning Framework 2030 [1]. It is the "ability to simply understand the other person's perspective [...] and to react to the observed experiences of another" (Davis 1983 [2], p.1), which is defined as empathy ${ }^{1}$. Empathy skills not only pave the foundation for successful interaction in digital companies, e.g., in agile work

1 Being aware that empathy is a multidimensional construct, in this study we focus on the emotional and cognitive empathy [2, 25]

16th International Conference on Wirtschaftsinformatik,

March 2021, Essen, Germany 
environments [3], but they are also one of the key abilities in the future that distinguish human work force and artificial intelligence agents [4]. However, besides the growing importance of empathy, research has shown that empathy skills of US college students have decreased from 1979 to 2009 by more than thirty percent and even more rapidly from 2000 to 2009 [5]. On these grounds, the Organization for Economic Cooperation and Development (OECD) claims that training empathy skills should receive a more prominent role in today's higher education [1]. To train empathy to students, educational institutions traditionally rely on experiential learning scenarios, such as shadowing, communication skills training or role-playing, e.g., in medical education [6]. Individual empathy training is therefore only available for a limited number of students, since individual tutoring through a student's learning journey is often hindered due to traditional large-scale lectures or the growing field of distance learning scenarios such as Massive Open Online Classes (MOOCs, [7]). However, to develop skills such as empathy, it is of great importance for the individual student to receive continuous feedback throughout their learning journey $[8,9]$. In fact, educational institutions are limited in providing these individual learning conditions especially for empathy skill training.

A promising way to support students to train the ability to react to other people's observed experiences [2] and enable teachers to convey it to classes of large sizes and independent from location might be the usage of adaptive technology-based applications in a pedagogical scenario for a student's learning journey (e.g., as done for other metacognition skills $[10,11])$. Researchers especially from the field of Educational Technology have designed pedagogical scenarios to train the empathy skills of students through virtual reality role-playing for social work education [12], virtual agents to simulate patient treatments for nurses (e.g., [6]) or adaptive empathy text feedback on computer-mediated communication platforms to foster empathy for company-client and employee-customer relationships [13].

However, novel technological-enhanced pedagogical scenarios based on recent advances of Natural Language Processing (NLP) or Machine Learning (ML) to design new forms of human-computer interaction for learners to train empathetic interaction through adaptive tutoring fall rather short in literature $[14,15]$. A possibility to provide adaptive empathy feedback on natural language bears the field of empathy detection form Computational Linguistics [16]. Empathy detection has been a growing research approach to identify and model empathetic structures and phrases of a given text in real-time, which could be leveraged to provide students with individual feedback, e.g., on peer reviews on business models or team conversation logs $[13,16]$. However, despite the vast amount of studies, current literature falls short of providing an approach with principles and proof on how to design an adaptive and intelligent learning tool to help students learn how to react to other students' perspectives with intelligent feedback on natural language. Thus, we aim to contribute to the field of technologyenhanced empathy learning by answering the following research question (RQ):

RQ: What are design principles for an adaptive learning tool that helps students to improve their empathy skill in large-scale or distance learning scenarios? 
To answer our research question, we follow the design science research approach (DSR) by Hevner (2007) [17]. As stated above, there is a lack of design knowledge for technology-enhanced tools to convey empathy skills. We intend to iteratively design and evaluate an IT learning artifact on the baseline of existing theory (cognitive dissonance based on Festinger (1962) [18]) informing the artifact design [19]. We believe cognitive dissonance theory could explain why formative text feedback on a student's empathy skills will motivate the student to be more aware and sensitive towards empathetic behavior. The theory has been widely applied in HCI and Information Systems (IS) research before, e.g., for adaptive argumentation skill learning (e.g., [11, 20]). To the best of our knowledge, there is no study that rigorously derives requirements from both scientific literature and potential users to develop an adaptive IT learning tool for helping IS students learn how to react to other students' perspectives based on this theory. With adaptive learning tool, we mean a tool which provides individual and real-time feedback on the emotional and cognitive empathy level to students on a given text, e.g., a chat conversation, and provides suggestions on how to write more empathetically, e.g., when writing peer reviews on business ideas. In this paper, we present our preliminary design principles we derive from literature and user interviews. Moreover, we provide a proof-of-concept evaluation of the instantiated design principles with 25 students. Our results indicate, that an adaptive empathy learning tool based on our design principles might be a promising approach to assist lecturer and educational organizations in helping students to receive empathy feedback on natural language input.

In the following, we will first introduce the reader to the necessary theoretical background. Afterwards, we present our methodological approach for developing the artifact following the three cycle view of Hevner (2007) [17]. We present our preliminary results, followed by a proof-of-concept evaluation. Finally, we discuss the results and close with a conclusion.

\section{Theoretical Background}

\subsection{Empathy Learning}

The ability to perceive the feelings of another person and to react to their emotions in the right way requires empathy - the ability "of one individual to react to the observed experiences of another" (Davis 1983 [2], p.1). Empathy plays an essential role in daily life in many practical situations, such as client communication, leadership or agile teamwork. Therefore, especially business schools today are increasingly trying to focus on fostering empathy skills [21] to provide students with the right skill set to meet future job profiles [22]. The importance of empathy and other metacognition skills has been manifested by the $O E C D$, which included them as a major element of their Learning Framework 2030 [1]. Despite the interdisciplinary research interest, the term empathy is defined from multiple perspectives in terms of its dimensions or components [23]. 
Being aware that there are multiple perspectives on empathy, in this paper we focus on the cognitive and emotional components of empathy as defined by Davis (1983) and Spreng et al. (2019) [2, 24]. Therefore, we follow the "Toronto Empathy Scale" [25] as a synthesis of instruments for measuring and validating empathy. Empathy refers to the "ability to simply understand the other person's perspective [...] and to react to the observed experiences of another" (Davis 1983 [2], p.1), where empathy consists of both emotional and cognitive components [25]. While emotional empathy lets us perceive what other people feel, cognitive empathy is the human ability to recognize and understand other individuals [24].

Besides the importance of empathy in daily life, studies have shown that empathy skills of US college students have decreased from 1979 to 2009 by more than thirty percent and even more rapidly in the last period from 2000 to 2009 [5]. Possible explanations are given by the growing amount of digital communication in our society [5]. Scientists therefore urge that training empathy skills should receive a more prominent role in today's higher education (e.g., [1, 12]). In fact, individual support of empathy learning is missing in most learning scenarios. In some domains training programs are designed to increase empathy skills through role plays, films, literature or video games (e.g., [21]). Since social professions, in particular, are characterized by interactions, similar training programs that promote empathy or empathetic forms of expression have so far also been successfully implemented for social workers [27], doctors and nurses [28]. In business education, empathy is usually trained through communication scenarios, classroom exercises, role plays or experiential learning (e.g., [21]). In fact, empathy is often regarded as a subcomponent of social competence [29], corresponding support measures often take place in extensive programs to promote social development.

However, in order to train skills such as empathy, it is essential for the individual student to receive continuous feedback, also called formative feedback, throughout the learning process [8]. According to Sadler (1989) [30], the result of feedback is specific information about the learning task or process that fills a gap between what is understood and what should be understood. Even in areas where empathy is part of the curriculum, such as health or social work, the ability of a teacher to provide tutoring is naturally limited by time and availability constraints. Especially in more frequent large lectures and distance learning scenarios, the ability to individually support a student's empathy ability is hampered because it is becoming increasingly difficult for educators to provide continuous and individual feedback to a single student.

\subsection{Technology-Based Learning Systems for Empathy Skills}

Many researchers, especially from the fields of Educational Technology, have analyzed how technology-based systems in sociotechnical scenarios can address this gap and enhance students' learning of empathy. The application of information technology in education bears several advantages, such as consistency, scalability, perceived fairness, widespread use and better availability compared to human teachers, and thus technology-enhanced empathy learning systems can help to relieve some of 
the burden on teachers to convey empathy by supporting learners with adaptive empathy feedback.

Scientist have successfully embedded computer-assisted instruction (CAI) in the form of virtual reality $(V R)$ learning tools in pedagogical scenarios to enable students to directly dive into the perspective of a peer, e.g., a client or patient (e.g., [28]). Moreover, intelligent tutoring systems (ITS) are used in the form of virtual agents built into online tools, e.g., to enable interaction with emotional avatars (e.g., [31]). Lastly, computer-supported collaborative learning (CSCL) tools are implemented to enhance empathy in the text communication of learners [13]. In their approach, Santos et al. (2018) [13] use a simple library of messengers based on neurolinguistics, psychometrics and text mining techniques to promote empathy among students' interaction, based on identification and text matching suggestions [13]. The combination of ITS and CSCL to design adaptive empathy learning tools is scarcely investigated in literature [13]. The aim is to provide pedagogical feedback on a learner's actions and solutions, hints and recommendations to encourage and guide future activities in the writing processes or automated evaluation to indicate whether a student's reaction to another person's perspective is emotionally appropriate. The design and implementation of ITS and CSCL to build adaptive learning tools is a rather complex endeavor that must rely on expertise from the fields of computer science (i.e., development of feedback algorithms), human-computer interaction (i.e., design of the interface) and educational technology (i.e., integration into the learning process).

Therefore, we aim to address this research gap and aim to contribute with rigorous design knowledge for an empathy learning tool based on educational theory through the application of recent developments in NLP and ML, in which empathy detection has been a growing research approach to identify and model empathetic structures of a given text in real-time $[13,16]$. The potential of empathy detection has been investigated in different domains but not leveraged for individual tutoring or feedback in a student's learning progress [16].

\subsection{Cognitive Dissonance as a Kernel Theory for Individual Learning}

We believe that Cognitive Dissonance Theory supports our underlying hypothesis that individual and personal feedback on a student's ability to react to other people's perspectives in an emotionally appropriate manner motivates the student to improve their skill level. Cognitive dissonance refers to the uncomfortable feeling that occurs when there is a conflict between one's existing knowledge or beliefs and contradicting presented information [18]. This unsatisfying internal state results in a high motivation to solve this inconsistency. According to Festinger's theory, an individual experiencing this dissonance has three possible ways to resolve it: change the behavior, change the belief or rationalize the behavior. Especially for students in a learning process, dissonance is a highly motivating factor to gain and acquire knowledge to actively resolve the dissonance [32]. It can be an initial trigger for a student's learning process and thus the construing of new knowledge structures [33]. However, the right portion of cognitive dissonance is very important for the motivation to solve it. According to Festinger, individuals might not be motivated enough to resolve it if the dissonance is 
too obvious, whereas a high level of dissonance might lead to frustration. Therefore, we believe that the right level of feedback on a student skill, such as empathy skills, could lead to cognitive dissonance and thus to motivation to change the behavior, belief or knowledge to learn how to react to other people's perspectives in an appropriate manner.

\section{$3 \quad$ Research Methodology}

Our research project is guided by the DSR approach [17]. Figure 1 shows the steps that are being carried out. We followed a theory-driven design approach by grounding our research on the cognitive dissonance theory [18].

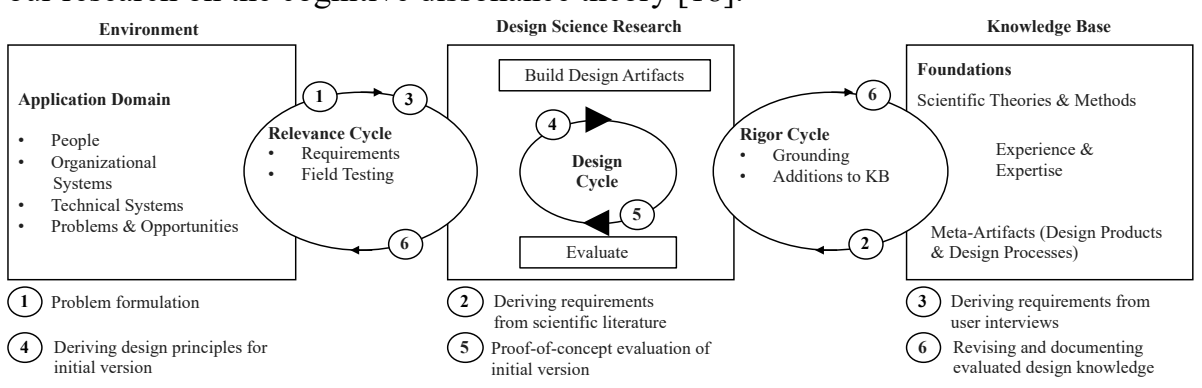

Figure 1. Three cycle design science process according to [17]

The first step of the DSR cycle includes the problem formulation. We therefore described the relevance of the practical problem in the introduction of this work. As the second step, we derived a set of meta-requirements (MRs) from the current state of scientific literature for the design of an empathy learning tool. Based on those insights, we conducted 28 semi-structured interviews with master students, using the expert interview method by Gläser and Laudel (2010) [34] and gathered user stories (USs) and user requirements (URs) for the design of an adaptive empathy learning tool based on those interviews. In the fourth step, we derived five preliminary design principles (DPs) addressing the MRs and URs using the structure suggested by Gregor et al. (2020) [35] and designed an initial version as a first instantiation of these DPs. In the fifth step, we conducted a proof-of-concept evaluation based on evaluation criteria proposed by Venable et al. (2016) [36]. Based on the design principles, we created a mock-up prototype, where students were able to receive an empathy feedback based on chosen pre-defined answers. The goal of this evaluation was to see how the students perceive the value of our instantiated design principles, to note change requests and to gather additional design principles. We conducted an experiment with 25 students to achieve our goal. The students had a short interaction with the prototype and received an adaptive empathy feedback based on chosen answers. Afterwards we captured their perception with a post-survey. In step six, we close with a short discussion thereby documenting the design knowledge. 


\section{Designing the Artifact}

In this section, we will describe and discuss how we gathered the preliminary requirements, derived the preliminary DPs and evaluated them in an instantiated initial version. The problem formulation (step one), described in the introduction, serves as the foundation for the derivation of the requirements. The main insights are illustrated in Figure 2.
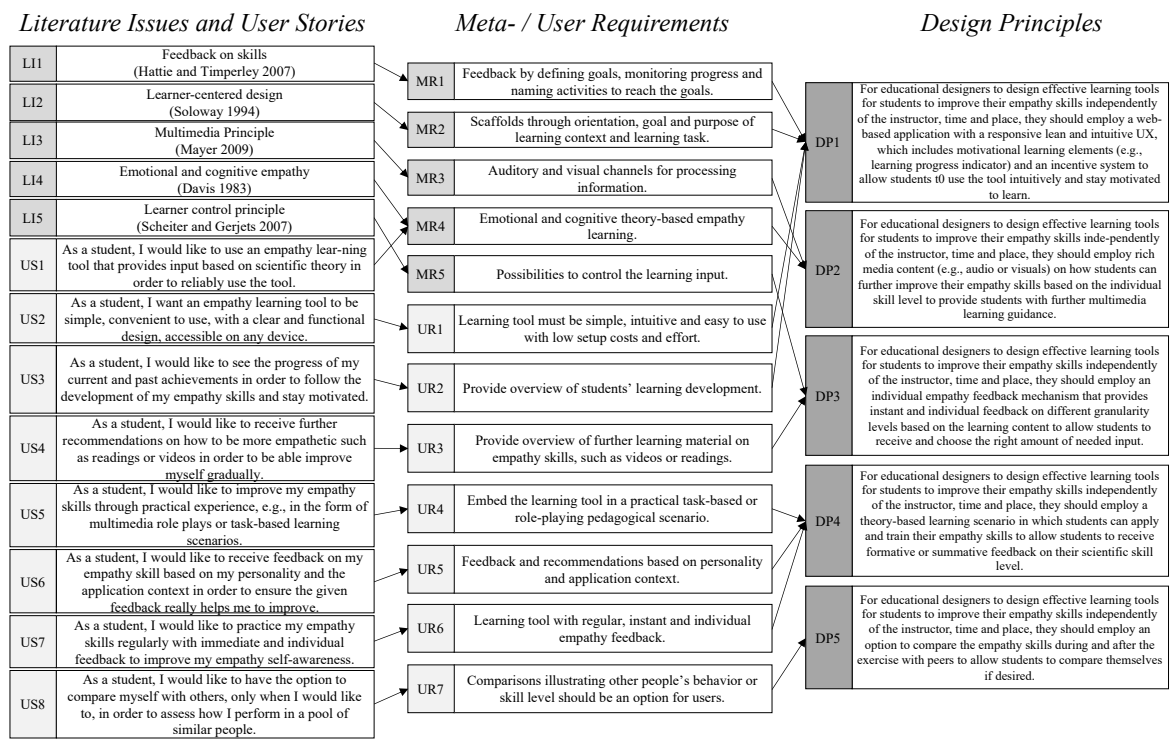

Figure 2. Overview of the derived design principles according to [35]

\section{Step 2: Deriving Meta-requirements from Scientific Literature}

To derive requirements from scientific literature, a systematic literature search was conducted using the methodological approaches of Cooper (1988) [37] and vom Brocke et al. (2015) [38]. We initially focused our research on studies that demonstrate the successful implementation of learning tools for empathy skills. Two broad areas for deriving requirements were identified: educational technology and learning theories. Since the creation of a learning tool for empathy skills is a complex project that is studied by psychologists, pedagogues and computer scientists with different methods, we first concentrated on these literature streams. We only included literature that deals with or contributes to a kind of learning tool in the field of empathy learning, such as an established learning theory.

On this basis, we selected 110 papers for more intensive analysis. We have summarized similar topics of these contributions as literature issues (LIs) and formed five clusters from them. Individual formative feedback is essential for the learning of skills such as empathy (LI1, i.e., [8]). Hence, it is crucial to define goals, monitor the 
progress towards the goals and name activities to reach the goals for the learner (MR1). Following their theory of learner-centered design (LI2), [39] named the concept of scaffolds with a specific goal, purpose and learning guidance as a central component of learning software when the purpose is to complete constructive activities such as writing empathetic texts (MR2). In his cognitive theory of multimedia learning, Mayer (2007) [40] named the "multimedia principles" (LI3), which states that "people learn more deeply from words and pictures than from words alone" (p.47, Mayer 2009). Therefore, to guide learners, the tool needs to incorporate both words and images to reduce the load for a single processing channel (MR3). Moreover, we follow the empathy construct of Davis (1983) [2] (LI4), which guides our empathy learning tool with the structure of emotional and cognitive empathy tutoring (MR4). Lastly, the learners' control principle (LI5) is of special significance for learning skills, since it aims to enable learners to adjust the information needed for their personal learning process (MR5) [41].

\section{Step 3: Deriving Requirements from User Interviews}

Based on the derived LIs and MRs, we conducted 28 semi-structured interviews according to Gläser and Laudel (2010) [34]. The interview guideline consists of 30 questions and each interview lasted mean $=40.91$ minutes $(\mathrm{S} D=15.9$ minutes $)$. The interviewees were a subset of students at our university who are all potential users of an empathy learning tool.

The participants were asked about the following topics: experience with technologybased learning systems, importance of skills in university education, requirements for a system that supports learning metacognition skills (e.g., functionalities, design) and requirements for a system that supports learning empathy (e.g., functionalities, design). In order to gain impressions resulting from many years of learning experience, only master students were recruited for the interviews. The interviewed students had a mean age of 24.82 years $(\mathrm{S} D=1.98)$ and all students were studying economics, law or psychology; 15 were male, 13 were female. After a more precise transcription, the interviews were evaluated using a qualitative content analysis. The interviews were coded, and abstract categories were formed. The coding was performed using open coding to form a uniform coding system. Based on these results, we gathered 269 user stories (USs) and identified seven user requirements (URs) following Cohn (2004) [42].

For all interviewees, it was very important that an empathy learning tool relies on a scientific theory to reliably use the tool (US1), which is reflected in MR4. All students mentioned that the learning tool must be simple, convenient to use, with a clear and functional design and accessible on any device (US2), which we incorporated in UR1. Moreover, all students stated that they would like to continuously use an empathy learning tool for practical experience, e.g., in the form of multimedia role plays or taskbased learning scenarios (US5, UR4), and therefore would like to see the progress of their learning development for current and past activities to stay motivated (US3, UR2). On top of that, a majority clearly mentioned that they would like to receive immediate and individual empathy feedback (US7, UR6) based on their personality and the 
context of the application scenario to ensure that the feedback is valuable for them (US6, UR5). The interviewed students also mentioned that they would like to receive direct recommendations on how to be more empathetic in a certain scenario and further learning material (e.g., readings or videos) to gradually improve themselves (US4, UR3). Regarding social comparison, we received differentiated feedback resulting that students would like to have an option to compare their individual empathy feedback with peers. The comparison function should not be directly shown without the user selecting it (US8, UR7).

\section{Step 4: Deriving Design Principles and Instantiating an Initial Version}

We have identified five LIs, eight USs and formulated five preliminary MRs and seven preliminary URs. Based on these findings, we derived five preliminary DPS following the structure of Gregor et al. (2020) [35] for an adaptive learning tool for empathy skills as a special class of learning tools for metacognition skills. The design principles are depicted in Figure 2.

Design principle 1 (DP1) states that the artifact should be developed as a web-based application with a responsive, lean, and adaptable user interface. The learning tool should also contain learning elements that motivate the students during the application. Therefore, we instantiated a lean and adaptive learning process with an intuitive learning experience and a dialogue-oriented interface. Furthermore, the student can learn with an individual empathy learning dashboard. The dashboard provides users with an intuitive overview of the learning content and empathy theory. Furthermore, feedback on the empathy task is displayed in different granularity levels, and further learning options are offered (e.g., comparison with other students). The dashboard also leads the user to a progress bar, which gives the student an overview of his learning progress, which underlines the motivating character of DP1. Besides, the learning tool is equipped with audio or visual material to provide the students with further multimedia learning support (DP2).

In DP4, we propose to use a learning-based scenario to train empathy. Therefore, the empathy learning tool should be embedded in a proven teaching-learning scenario that is easy to set up and domain-agnostically applicable. We used student peer reviews, as students can apply and train their empathy skills by giving feedback on a peer's business model [39]. The potential of student peer reviews and training of metacognition skills has already been successfully demonstrated for other NLP-based skill training, such as argumentation skills [40]. DP5 includes the possibility for students to compare their empathy levels with other students. One way to do this could be a progress bar, which allows students to compare themselves optionally.

Next, DP3 emphasizes the need for individual feedback to learn skills such as empathy. Students should receive feedback on the pre-defined response options that have been selected beforehand. Therefore, we have introduced a direct and individual feedback mechanism to help students train their empathy skills. We also set up a mechanism to provide students with further learning material. The advanced learning material consists of videos and literature. These materials will help students to learn more about the different dimensions of empathy. 
To instantiate and evaluate the design principles above, we created a mock-up-based prototype by using the tool marvel $^{2}$. Our prototype (Figure 3) guides students through providing a peer review on another student's business model through a conversational interface and an empathy learning dashboard. Our DPs were formulated based on the analysis of current issues related to the theory of learning and teaching metacognition skills and needs and requirements of users based on cognitive dissonance theory [18]. We argue that a learning tool for empathy skills (and possibly other metacognition skills) that instantiates our DPs should increase the motivation of students to learn how to apply the certain skills, for example, learn how to appropriately react to another person's perspective and thus improve the learning outcome. For example, an empathy learning tool that provides instant and individual feedback and gives students the flexibility to control their learning input and provides further learning material should increase the students' motivation to resolve dissonance and therefore construct new knowledge.

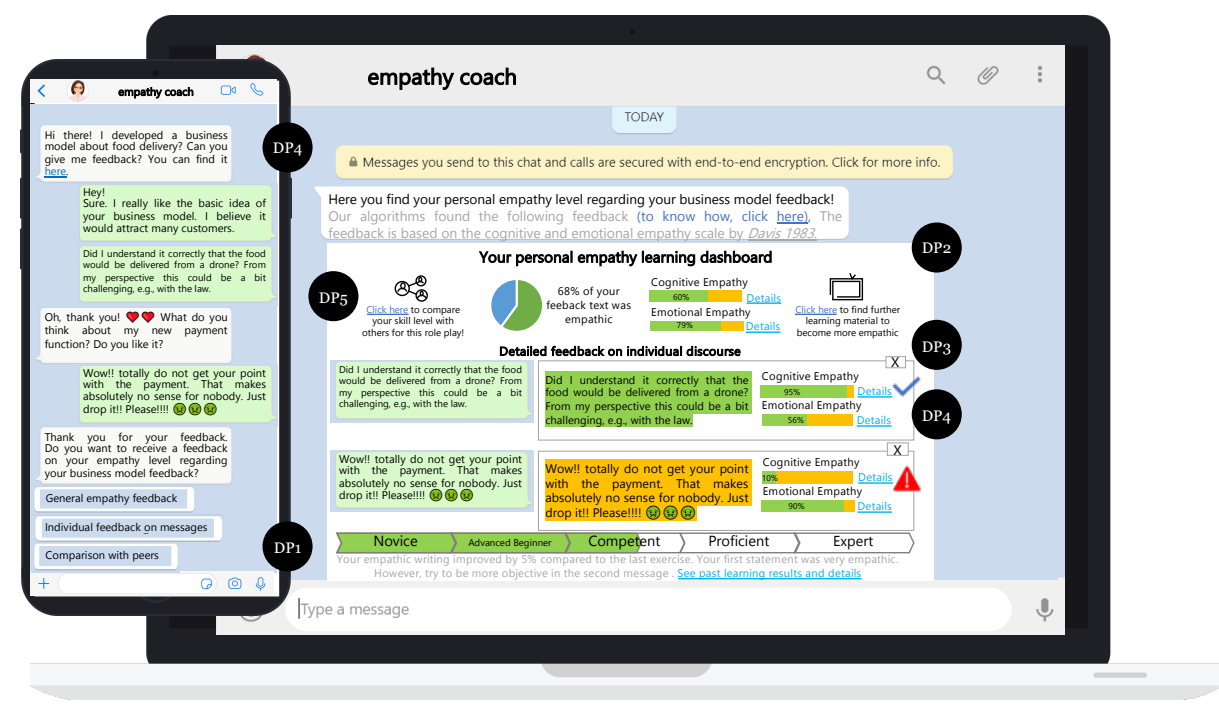

Figure 3. Empathy learning tool based on the preliminary design principles (DP 1-5)

\section{Proof-of-Concept Evaluation of Initial Version}

In this section, we describe the proof-of-concept evaluation of the initial version of our empathy learning tool. Based on the gathered requirements and design principles, we designed a clickable mock-up displaying a conversational learning interface which provides empathy feedback based on pre-defined answers (without implementing trained chat intents in the back end). For the evaluation, we followed an ex ante evaluation using an artificial evaluation setup as proposed by Venable et al. (2016) [36]. The purpose of the evaluation is to check whether the design principles are useful

2 marvelapp.com 
for learners, in order to incorporate any change requests. The design principles were specifically examined based on the criteria of usefulness and usability and evaluated by means of various questions. Therefore, a questionnaire with 14 items was created with specific questions about the DP, e.g., we asked questions about the perception of the empathy learning input, the usability and usefulness of the adaptive empathy functions and the concept of the adaptive learning tool as a whole.

To do so, we designed an experiment in which participants were asked to provide a peer review based on a provided business model essay. The participants were using our initial version for providing a business model review to an imaginary peer (see Figure 3). After the review task, they received adaptive feedback on their cognitive and their emotional empathy level based on Davis (1983) [2]. Afterwards, we asked specific question to evaluate the design principles. Thus, we gave participants items addressing the instantiated principles: For evaluating DP1, „The learning journey would give me an overview of my learning process and thus motivate me. "; for DP2, "I would find the information about learning empathy helpful."; for DP3, "The rating of my messages reflects my actual empathy.", "The tool has accurately rated my empathy" and "The feedback I received from the tool was an accurate rating of my empathy".; for DP4, "I assume that the learning tool would help me improve my ability to give empathically appropriate feedback." and "I assume that the learning tool would help me improve my ability to give emotionally empathically appropriate feedback."; and for DP5, "I would find the possibility to compare my empathy level with others useful." All answers were captured on a 1-to 5-point Likert scale (1: totally disagree to 5: totally agree, with 3 being a neutral statement). Additionally, we asked three qualitative questions: "What did you particularly like about the use of the empathy tool?", "What else could be improved?" and "Do you have any other ideas?" to both groups. Finally, we captured the demographics.

Table 1. Overview of the results of the proof-of-concept evaluation of our design principles

\begin{tabular}{|c|c|c|c|c|c|}
\hline $\mathrm{n}=25$ & DP1 & DP2 & DP3 & DP4 & DP 5 \\
\hline mean & 3.64 & 3.5 & 2.9 & 3.5 & 3.8 \\
\hline SD & 0.86 & 0.87 & 0.73 & 0.88 & 0.76 \\
\hline normalized & 0.72 & 0.7 & 0.58 & 0.7 & 0.76 \\
\hline
\end{tabular}

In total, we received 25 completed answers. 17 were from males, 8 from females. The mean age was 25.12 years $(\mathrm{SD}=2.78)$. Our evaluation confirmed that DP1, DP2, DP4 and DP5 are mostly positively perceived by the participants (see Table 1). The mean values for the DPs are promising when comparing the results to the midpoints of the scale. The results for DP1, DP2, DP4 and DP5 are better than the neutral value of 3 and all normalized values are equal or greater than 0.7. Only the rating of DP3 is less positive with a neutral value of 2.9 (see Table 1).

We also included open questions in the survey to get students' impressions of how they perceived their interaction with our initial version and further evaluate DP. The respondents were asked to indicate what they liked, what weaknesses they see, and 
whether they had any ideas for improving the tool. The general attitude towards interaction with our tool was very positive. Data analysis confirms that students are interested in using a learning tool for empathy skills and would be motivated to work with it. A learning tool that evaluates empathy is perceived as "very useful". Participants emphasized, however, that "the tool was easy to use. The fun factor was also present, and it was fun to write with the bot" (DP1). They also expressed their confidence in the instrument and praised the theoretically well-founded background, which "explains the different types of empathy in more detail" (DP2). The direct and individual feedback and the resulting potential for improvement for users were mentioned by many participants (DP3), e.g.: "The tool obviously and objectively evaluates a skill that previously seemed subjective to me. This helps to improve oneself better and to identify possible improvement potentials". The qualitative evaluation also revealed some interesting and relevant suggestions for improvement. The participants asked for more pre-defined response options for the business model feedback in order to be able to give specific answers. Also, the pre-defined response options should be more differentiated. Many also mentioned that they would like to write the feedback themselves in natural text, e.g. the Toll should be equipped with an "extended answer function" to get "more individual feedback" on the answers (DP3).

\section{Discussion and Conclusion}

In this paper, we derived a set of five preliminary design principles on how to design an adaptive empathy learning tool. Therefore, we discussed five literature issues based on 110 scientific papers and presented five preliminary MRs and seven URs from 28 interviews. We built an initial version as an instantiation of these design principles, evaluated the principles through our initial version in a proof-of-concept evaluation [36] and captured the perception of students.

Therefore, our work makes several contributions to research. To the best of our knowledge, we provide the first study with evaluated design principles for the design of an empathy learning tool. Our DPs were formulated based on the analysis of current issues related to theories of learning and teaching metacognition skills and needs and requirements of users based on cognitive dissonance theory [18]. We argue that a learning tool for empathy skills (and possibly other metacognition skills) that instantiates our DPs should increase the motivation of students to learn how to apply certain skills, for example, learn how to appropriately react to another person's perspective and thus improve the learning outcome. For example, an empathy learning tool that provides instant and individual feedback and gives students the flexibility to control their learning input and provides further learning material should increase the students' motivation to resolve dissonance and therefore construct new knowledge. We argue that lecturers and educational institutions can use these design principles to create their own empathy learning tools to improve their individual pedagogical scenarios. Our evaluation showed that the initial design principles are promising for students to use such a learning tool. Only DP3 falls short on expectations in our data analysis. However, we believe, that the relatively low ratting of the items is related to the mock- 
up version of our prototype with only predefined response options. Since the answer options do not reflect the students' individual empathy level, the feedback from the students is not seen as corresponding to their personal empathy level. By extending the tool with a function that allows users to write personal answers, we think we might be able to resolve the discrepancy in the evaluation of DP3.

A number of limitations have to be considered with respect to our study. First, we gathered requirements from a certain theoretical perspective and a specific user group. It might be possible that other areas of literature and user groups might have led to different results. Moreover, we were not yet able to fully implement our empathy learning tool with a fully functional automatic feedback algorithm based on NLP and ML in the back end (reflected in responses towards DP3). In fact, we are creating a new annotation scheme (such as [43] for argumentation skills) to capture emotional and cognitive empathy structures in student peer feedbacks with the aim to train a predictive model to provide students with individual skill feedback based on deep and transfer learning $[15,44]$. Therefore, we aim to analyze the impact of the instantiated learning tool on students' learning performance in a large-scale lecture experiment in the future. The trained model could be also embedded in a conversational tutoring system, e.g., to enhance user satisfaction in education such as $[14,45,46]$. We expect our overall research project to contribute a nascent design theory [47] to the artifact class of IT learning tools for metacognition skills and thus contribute to the OECD Learning framework 2030 towards a metacognition-skill-based education.

\section{References}

1. OECD: The Future of Education and Skills - Education 2030, (2018). https://doi.org/201806-15.

2. Davis, M.H.: Measuring individual differences in empathy: Evidence for a multidimensional approach. J. Pers. Soc. Psychol. 44, 113-126 (1983). https://doi.org/10.1037//00223514.44.1.113.

3. Luca, J., Tarricone, P.: Does Emotional Intelligence Affect Successful Teamwork? Proc. 18th Annu. Conf. Australas. Soc. Comput. Learn. Tert. Educ. 367-376 (2001).

4. Poser, M., Bittner, E.A.C.: Hybrid Teamwork: Consideration of Teamwork Concepts to Reach Naturalistic Interaction between Humans and Conversational Agents. In: WI2020. GITO Verlag (2020). https://doi.org/10.30844/wi_2020_a6-poser.

5. Konrath, S.H., O'Brien, E.H., Hsing, C.: Changes in dispositional empathy in American college students over time: A meta-analysis. Personal. Soc. Psychol. Rev. 15, 180-198 (2011). https://doi.org/10.1177/1088868310377395.

6. Lok, B., Foster, A.E.: Can Virtual Humans Teach Empathy? In: Teaching Empathy in Healthcare. pp. 143-163. Springer International Publishing (2019). https://doi.org/10.1007/978-3-030-29876-0_9.

7. Seaman, J.E., Allen, I.E., Seaman, J.: Higher Education Reports - Babson Survey Research Group. (2018).

8. Hattie, J., Timperley, H.: The Power of Feedback. Rev. Educ. Res. 77, 81-112 (2007). https://doi.org/10.3102/003465430298487. 
9. Vygotsky, L.S.: Mind in society: The development of higher psychological processes. Harvard university press (1980).

10. Wambsganss, T., Söllner, M., Leimeister, J.M.: Design and Evaluation of an Adaptive Dialog-Based Tutoring System for Argumentation Skills. In: International Conference on Information Systems (ICIS). , Hyderabad, India (2020).

11. Wambsganss, T., Rietsche, R.: Towards designing an adaptive argumentation learning tool. In: 40th International Conference on Information Systems, ICIS 2019. pp. 1-9. , Munich, Germany (2020).

12. Gerdes, K.E., Segal, E.A., Jackson, K.F., Mullins, J.L.: Teaching empathy: A framework rooted in social cognitive neuroscience and social justice. J. Soc. Work Educ. 47, 109-131 (2011). https://doi.org/10.5175/JSWE.2011.200900085.

13. Santos, B.S., Junior, M.C., De Souza, J.G.: An Experimental Evaluation of the NeuroMessenger: A Collaborative Tool to Improve the Empathy of Text Interactions. Proc. - IEEE Symp. Comput. Commun. 2018-June, 573-579 (2018). https://doi.org/10.1109/ISCC.2018.8538442.

14. Zierau, N., Wambsganss, T., Janson, A., Schöbel, S., Leimeister, J.M.: The Anatomy of User Experience with Conversational Agents: A Taxonomy and Propositions of Service Clues. In: International Conference on Information Systems (ICIS). pp. 1-17 (2020).

15. Landolt, S., Wambsganss, T., Matthias, S.: A Taxonomy for Deep Learning in Natural Language Processing. In: Hawaii International Conference on System Sciences (HICSS) (2021).

16. Buechel, S., Buffone, A., Slaff, B., Ungar, L., Sedoc, J.: Modeling empathy and distress in reaction to news stories. Proc. 2018 Conf. Empir. Methods Nat. Lang. Process. EMNLP 2018. 4758-4765 (2018). https://doi.org/10.18653/v1/d18-1507.

17. Hevner, A.R.: A three cycle view of design science research. Scand. J. Inf. Syst. 1-6 (2007).

18. Festinger, L.: Cognitive Dissonance. Sci. Am. 207, 93-106 (1962). https://doi.org/10.1038/scientificamerican1062-93.

19. Hevner, A.R., March, S.T., Park, J., Ram, S.: Design Science in Information Systems Research. Des. Sci. IS Res. MIS Q. 28, 75 (2004).

20. Wambsganss, T., Niklaus, C., Cetto, M., Söllner, M., Leimeister, J.M., Handschuh, S.: AL : An Adaptive Learning Support System for Argumentation Skills. In: ACM CHI Conference on Human Factors in Computing Systems. pp. 1-14 (2020).

21. Peterson, R.T., Limbu, Y.: The convergence of mirroring and empathy: Communications training in business-to-business personal selling persuasion efforts. J. Business-to-bus. Mark. 16, 193-219 (2009). https://doi.org/10.1080/10517120802484551.

22. vom Brocke, J., Maaß, W., Buxmann, P., Maedche, A., Leimeister, J.M., Pecht, G.: Future Work and Enterprise Systems. Bus. Inf. Syst. Eng. 60, 357-366 (2018). https://doi.org/10.1007/s12599-018-0544-2.

23. Decety, J., Jackson, P.L.: The functional architecture of human empathy., (2004). https://doi.org/10.1177/1534582304267187.

24. Lawrence, E.J., Shaw, P., Baker, D., Baron-Cohen, S., David, A.S.: Measuring empathy: Reliability and validity of the Empathy Quotient. Psychol. Med. 34, 911-919 (2004). https://doi.org/10.1017/S0033291703001624.

25. Spreng, R.N., McKinnon, M.C., Mar, R.A., Levine, B.: The Toronto empathy questionnaire: Scale development and initial validation of a factor-analytic solution to multiple empathy 
measures. J. Pers. Assess. 91, 62-71 (2009). https://doi.org/10.1080/00223890802484381.

26. Bell, H.: Creative Interventions for Teaching Empathy in the Counseling Classroom. J. Creat. Ment. Heal. 13, 106-120 (2018). https://doi.org/10.1080/15401383.2017.1328295.

27. Hen, M., Goroshit, M.: Emotional competencies in the education of mental health professionals. Soc. Work Educ. 30, 811-829 (2011). https://doi.org/10.1080/02615479.2010.515680.

28. Bailenson, J.N., Yee, N., Blascovich, J., Beall, A.C., Lundblad, N., Jin, M.: The use of immersive virtual reality in the learning sciences: Digital transformations of teachers, students, and social context, (2008). https://doi.org/10.1080/10508400701793141.

29. Weis, S., Süß, H.-M.: Social Intelligence-A Review and CriticalDiscussion of Measurement Concepts. In: Schulze, R. and Roberts, R.D. (eds.) Emotional intelligence.An international handbook. pp. 203-230. Hogrefe (2005).

30. Sadler, D.R.: Formative assessment and the design of instructional systems. Instr. Sci. 18, 119-144 (1989). https://doi.org/10.1007/BF00117714.

31. Kralicek, D., Von Rabenau, L., Shelar, S., Blikstein, P.: Inside out:Teaching empathy and social-emotional skills. In: IDC 2018 - Proceedings of the 2018 ACM Conference on Interaction Design and Children. pp. 525-528. Association for Computing Machinery, Inc, New York, NY, USA (2018). https://doi.org/10.1145/3202185.3213525.

32. Elliot, A.J., Devine, P.G.: On the motivational nature of cognitive dissonance: Dissonance as psychological discomfort. J. Pers. Soc. Psychol. 67, 382-394 (1994). https://doi.org/10.1037/0022-3514.67.3.382.

33. Piaget, J., Brown, T., Thampy, K.J.: The Equilibration of Cognitive Structures: The Central Problem of Intellectual Development. Jean Piaget, Terrance Brown, Kishore Julian Thampy. Am. J. Educ. 94, 574-577 (1986). https://doi.org/10.1086/443876.

34. Gläser, J., Laudel, G.: Experteninterviews und qualitative Inhaltsanalyse : als Instrumente rekonstruierender Untersuchungen. VS Verlag für Sozialwiss (2010).

35. Gregor, S., Chandra Kruse, L., Seidel, S.: The Anatomy of a Design Principle. J. Assoc. Inf. Syst. Forthcomin, (2020).

36. Venable, J., Pries-Heje, J., Baskerville, R.: FEDS: A Framework for Evaluation in Design Science Research. Eur. J. Inf. Syst. 25, 77-89 (2016). https://doi.org/10.1057/ejis.2014.36.

37. Cooper, H.M.: Organizing knowledge syntheses: A taxonomy of literature reviews. Knowl. Soc. 1, 104-126 (1988). https://doi.org/10.1007/BF03177550.

38. vom Brocke, J., Simons, A., Riemer, K., Niehaves, B., Plattfaut, R., Cleven, A.: Standing on the shoulders of giants: Challenges and recommendations of literature search in information systems research. Commun. Assoc. Inf. Syst. 37, 205-224 (2015). https://doi.org/10.17705/1cais.03709.

39. Soloway, E., Guzdial, M., Hay, K.E.: Learner-Centered Design: The Challenge for HCI in the 21st Century. interactions. 1, 36-48 (1994). https://doi.org/10.1145/174809.174813.

40. Mayer, R.E.: Multimedia Learning. Cambridge University Press, Cambridge (2009). https://doi.org/10.1017/CBO9780511811678.

41. Scheiter, K., Gerjets, P.: Learner control in hypermedia environments. Educ. Psychol. Rev. 19, 285-307 (2007). https://doi.org/10.1007/s10648-007-9046-3.

42. Cohn, M.: User Stories Applied For Agile Software Development. (2004).

43. Wambsganss, T., Niklaus, C., Söllner, M., Handschuh, S., Leimeister, J.M.: A Corpus for Argumentative Writing Support in German. In: 28th International Conference on 
Computational Linguistics (Coling) (2020).

44. Wambsganss, T., Molyndris, N., Söllner, M.: Unlocking Transfer Learning in Argumentation Mining: A Domain-Independent Modelling Approach. In: 15th International Conference on Wirtschaftsinformatik., Potsdam, Germany (2020). https://doi.org/10.30844/wi_2020_c9wambsganss.

45. Wambsganss, T., Winkler, R., Schmid, P., Söllner, M.: Unleashing the Potential of Conversational Agents for Course Evaluations: Empirical Insights from a Comparison with Web Surveys. In: Twenty-Eighth European Conference on Information Systems (ECIS2020). pp. 1-18. , Marrakesh, Morocco (2020).

46. Wambsganss, T., Winkler, R., Schmid, P., Söllner, M.: Designing a Conversational Agent as a Formative Course Evaluation Tool. In: 15th International Conference on Wirtschaftsinformatik., Potsdam, Germany (2020).

47. Gregor, S., Hevner, A.R.: Positioning and Presenting Design Science Research for Maximum Impact. (2013). 